\title{
Evaluación de los riesgos psicosociales con el ISTAS-21
}

María Teresa Alonzo Castillo, María Estefanía Martínez Morillas y Cristina Molina Hidalgo. Universidad de Granada

Recepción: 12 de mayo de 2015 | Revisión: 17 de junio de 2015 | Aceptación/Publicación: 17 de julio de 2015

Citar: Alonzo, M.T., Martinez, M.E. y Molina, C. (2015). Evaluación de los riesgos psicosociales con el ISTAS-21. ReiDoCrea, 4 $123-128$.

Resumen: Presentamos un proceso de evaluación de los seis grandes grupos de factores de riesgos psicosociales que pueden afectar la salud en el trabajo, en el caso de un hombre de 47 años de edad. El instrumento de evaluación utilizado es la versión corta del Cuestionario de Evaluación de Riesgos Psicosociales en el Trabajo ISTAS21 (CoPsoQ) en el que permite evaluar e identificar los riesgos, además de facilitar el diseño e implementación de medidas preventivas. En este caso el sujeto sacó puntuaciones significativas en tres de estos grandes grupos de factores de riesgo psicosociales, los cuales son: exigencias psicológicas, doble presencia y estima. También presentamos una posible intervención terapéutica reducir o eliminar estos riesgos psicosociales que afectan en la salud del sujeto.

Palabras clave: riesgos psicosociales, trabajo, exigencias psicológicas, doble presencia y estima.

\section{Introducción}

Los riesgos psicosociales son características de las condiciones de trabajo y de la organización del trabajo, nocivas para la salud. Nuestro sujeto presenta una puntuación significativa en uno de los cuatro grandes grupos de riesgos psicosociales, en exigencias psicológicas. Concretamente presenta un exceso de exigencias psicológicas del trabajo; hay que trabajar rápido o de forma irregular, el trabajo requiere que escondamos los sentimientos... (ISTAS, 2002). La literatura previa define las exigencias psicológicas como todos aquellos aspectos sociales $u$ organizativos del trabajo que requieren un esfuerzo psicológico en el trabajador y, por ello, están asociados a cargas psicológicas, como por ejemplo la tensión, el estrés o la ansiedad (Mauno et al., 2007).

Las exigencias psicológicas del trabajo pueden ser de tipo cuantitativo, cuando se refieren al volumen del trabajo con relación al tiempo del que se dispone para llevarlo a cabo; o de tipo cualitativo, cuando hablamos de las demandas emocionales, cognitivas o sensoriales que suponen el desarrollo del trabajo. Según el Instituto Sindical de Trabajo, Ambiente y Salud (2003), las exigencias psicológicas tienen una doble vertiente: cuantitativa y cualitativa.

Desde el punto de vista cuantitativo, las exigencias psicológicas se refieren al volumen de trabajo con relación al tiempo disponible para hacerlo.

Desde el punto de vista cualitativo, las exigencias psicológicas se refieren a las demandas emocionales (cuando el trabajo expone a las personas a procesos de transferencia de emociones y sentimientos, cuando requiere esconder sentimientos y opiniones), cognitivas (cuando el trabajo requiere gran esfuerzo intelectual) 0 sensoriales (cuando requiere esfuerzo de los sentidos).

Demasiadas exigencias sean del tipo que sean son perjudiciales para la salud (Instituto Sindical de Trabajo, Ambiente y Salud, 2003). Así, la sociedad de hoy en día, se enfrenta a un nuevo escenario complejo en el que más allá de los roles de género, hombres y mujeres se enfrentan al reto de levantarse cada día y buscar el modo de 
atender a sus responsabilidades laborales y familiares, lo que se conoce en el cuestionario versión corta "Instas 21" como "doble presencia". Este contexto viene producido por varios factores, entre ellos por los cambios económicos y sociales de las últimas décadas (lo que ha hecho que las empresas establezcan en muchos casos turnos interminables en el trabajo), además de la incorporación de la mujer al mundo laboral, haciendo que quede desdibujada la imagen en la que el hombre se dedica exclusivamente al trabajo y la mujer al cuidado del hogar (Moreno-Jiménez y Garrosa, 2013).

El entorno familiar puede ser una fuente de estrés, además de ser considerado el principal antecedente de conflicto familia-trabajo. Especialmente el número de hijos en edad preescolar dificulta compaginar estos dos ámbitos. Los problemas de pareja también pueden generar preocupaciones que dificultan el desempeño de las tareas laborales al centrar los pensamientos en asuntos no relacionados con el trabajo. Esto a su vez conlleva a consecuencias importantes en la vida privada de las personas. En cuanto a las consecuencias organizacionales, destacan principalmente las intenciones de abandono y el absentismo. Por su parte, en los efectos familiares, la mayoría de estudios sobre conflicto han tratado de demostrar cómo la falta de equilibrio entre estos dos ámbitos conduce a mayores niveles de insatisfacción familiar. Cuando una persona experimenta conflicto, significa que no puede dedicar el tiempo o energía que le gustaría a otras actividades por lo que el nivel de satisfacción con varios aspectos de su vida disminuye. Además, la salud y el bienestar de las personas se ven afectados ante las dificultades para compaginar trabajo y familia, disminuyendo el bienestar físico y psicológico, la calidad de sueño e incluso la satisfacción con la vida (Moreno-Jiménez y Garrosa, 2013).

Para la disminución de todas estas implicaciones que afectan a las personas que intentan conciliar la vida laboral y familiar, podemos encontrar una serie de variables moderadoras. A nivel organizacional, se encuentran las políticas de conciliación en su empresa. Contar con la familia o una pareja que comprenda la situación laboral proporcionando comprensión y apoyo a nivel tanto instrumental como emocional, hará más probable que la experiencia de conflicto disminuya. También son importantes las características de la personalidad, así como saber gestionar sus emociones y desarrollar sus fortalezas para eliminar la "doble presencia" en su día a día (MorenoJiménez y Garrosa, 2013).

Por otro lado, decir que todas las personas tienen seis aspectos para la formación de su yo: lo que son, lo que piensan que son, lo que piensan otras que son, lo que creen que piensan las demás personas de ellas, lo que quieren llegar a ser y las expectativas que creen que otras personas tienen sobre ellas (Rice, 2000).

Strang, citado por Rice (2000), propone la existencia de cuatro dimensiones básicas del yo. En primer lugar el autoconcepto básico, que es la visión que la persona tiene sobre su personalidad y las percepciones de sus habilidades, su estatus y sus funciones en el mundo exterior. Posteriormente, tendríamos los autoconceptos temporales o transitorios. Estas ideas sobre la propia persona van a estar influenciadas por el estado de ánimo del momento o por una experiencia reciente. Por ejemplo, que tu jefe menosprecie el trabajo que has realizado puede hacer que la persona se sienta temporalmente como poco capaz, de forma que una crítica aguda por parte del otro puede producir un sentimiento transitorio de una autoestima devaluada.

En tercer lugar, encontramos el yo social, esto se refiere a la opinión que las personas creen que otras personas tienen sobre ellas, que a su vez, influye en ellas mismas. De 
forma que si tienen la impresión de que otros piensan que ellas son incapaces o socialmente inaceptables, pensarán sobre sí mismas de forma negativa. La cuarta dimensión es el yo ideal, es decir, qué tipo de personas les gustaría ser. Las aspiraciones pueden ser realistas, demasiado bajas o demasiado altas. Los yo ideales demasiado bajos impiden la realización, mientras que los demasiados altos pueden llevar a frustraciones o autodesprecio. Los conceptos del yo realistas conducen a la autoaceptación, la salud mental y la consecución de metas realistas (Rice, 2000).

El término autoestima se refiere a la confianza básica en las propias potencialidades, y apunta a dos componentes esenciales: La valía personal y el sentimiento de capacidad personal (Undurraga \& Avendaño, 1998). La valía personal es la valoración positiva o negativa que la persona tiene de su autoconcepto (imagen de sí mismo), incluyendo las emociones asociadas con esta valoración y las actitudes respecto de sí mismo. El sentimiento de capacidad personal alude a las expectativas que tiene una persona de ser capaz, de hacer de manera exitosa lo que tiene que hacer, es decir, su autoeficacia (FOCUS on Young Adults, 1998).

Alcántara, en Molina, Baldares y Maya (1996), habla de la autoestima no como algo innato, sino algo que se adquiere y se genera como resultado de la historia de cada persona. Este aprendizaje generalmente no es intencional, por cuanto se moldea a la persona desde diversos contextos informales.

Un factor importante a tener en cuenta es que la autoestima está configurada tanto por factores internos como externos. Entendiendo por factores internos, los que radican y son creados por el propio individuo, es decir, sus ideas, creencias, prácticas y conductas. $Y$ entendiendo por factores externos aquellos pertenecientes al entorno, tales como, los mensajes transmitidos verbal o no verbalmente, las experiencias suscitadas por los padres, educadores, personas significativas para nosotros, compañeros, las organizaciones y la propia cultura (Branden, 2004). Es desde esta perspectiva cualitativa, desde la que pueden afectar las exigencias psicológicas del trabajo a las personas. Pues la autoestima se desarrolla a partir de la interacción humana, a partir de la cual unas personas se consideran importantes para las otras. El yo va evolucionando por medio de pequeños logros, reconocimientos y éxitos (Tarazona, 2014).

\section{Método}

\section{Instrumento, procedimiento y participante}

El cuestionario elegido para analizar los riesgos psicosociales de un sujeto ha sido la versión corta para pequeñas empresas y autoevaluación del cuestionario Instas21, una versión en lengua castellana del cuestionario psicosocial de Copenhague (COPSOQ). Este instrumento se usa para identificar y cuantificar la exposición a 6 grupos de factores de riesgo para la salud psicosocial en el ámbito del trabajo, los cuales son: exigencias psicológicas, trabajo activo y posibilidades de desarrollo (influencia, desarrollo de habilidades y control sobre los tiempos), apoyo social y calidad de liderazgo, inseguridad, doble presencia y estima. Los valores en los que se sitúa el participante se clasifican en verde (nivel de exposición psicosocial más favorable para la salud), amarillo (nivel de exposición psicosocial intermedio) o rojo (nivel de exposición psicosocial más desfavorable para la salud), en función de su puntuación total en cada uno de los grupos.

Para seleccionar al participante no se hecho ningún proceso de selección o de forma azarosa, sino que se buscó a un profesor de la facultad sin que tuviera que tener 
ningún prerrequisito. Una vez resuelto el cuestionario, se procesa a calcular la puntuación, tal y como indica el cuestionario, a clasificar cada una de las dimensiones en valores de rojo, amarillo y verde. A continuación, se debate con el participante cual cree que son los motivos por los que ha puntuado en riesgo psicosocial en las dimensiones en rojo.

Este cuestionario ha sido pasado en la facultad de psicología de Granada, en la clínica de psicología, el día 10 de marzo de 2015. Ha sido rellenado por un hombre de 46 años, profesor de la facultad de psicología del departamento de personalidad, evaluación y tratamiento psicológico. El participante tiene de 15 a 20 años de antigüedad en el puesto, además de su trabajo como profesor, trabaja en la clínica de la facultad, realiza investigaciones y participa en un grupo político. Actualmente tiene un hijo de 12 años y está casado.

\section{Resultados}

Los resultados que se obtuvieron tras pasar el cuestionario fueron los que aparecen en la siguiente tabla:

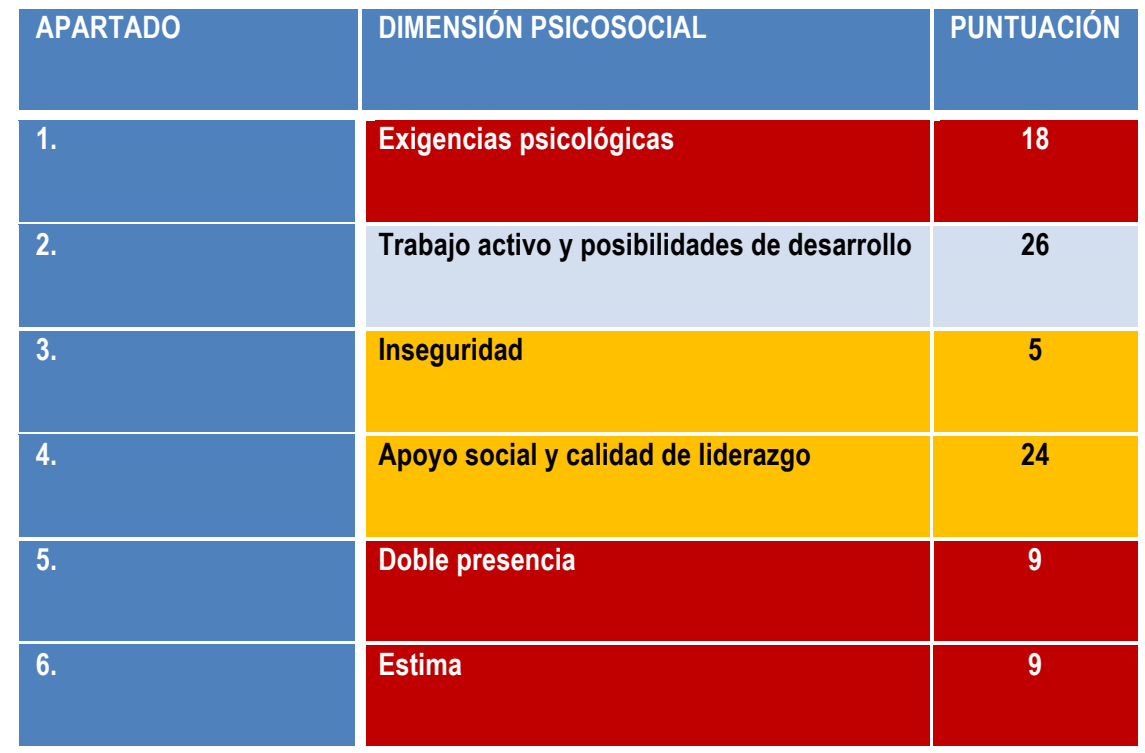

Como se puede observar en la tabla anterior encontramos tres dimensiones en rojo, dos en amarillo, en las que no nos centraremos y tan solo una en azul, que tampoco es de nuestro interés. En cuanto a las dimensiones en rojo, se traduce que la persona a la que se le pasó el cuestionario tiene puntuaciones más desfavorables para su salud en las dimensiones de: Exigencias psicológicas (puntuación entre 11 y 24); Doble presencia (puntuación entre 7 y 16) y Estima (puntuación menor a 10), situándose así entre la población ocupada que peor está en cuanto a exigencias psicológicas del trabajo, en cuanto a compaginar vida laboral y familiar, y en cuanto a la forma en la que él percibe que los demás le ven y valoran.

\section{Discusión}

En vista a las puntuaciones que ha obtenido nuestro participante, podemos concluir que al encontrarse en tres de las seis dimensiones en rojo, las consecuencias que se derivan de sus condiciones de trabajo pueden tener consecuencias negativas en su 
salud. Es más, hoy por hoy, dichas consecuencias podrían manifestarse en forma de malestar físico y mental.

En cuanto a las exigencias psicológicas, las causas de las que deriva su situación, podrían venir determinadas por el horario, siendo este muy irregular y abarcando todo su día, una cantidad alta de trabajo que hacer, la sensación de no poder decir que no al trabajo que se le exige, además de un cúmulo de exigencias por parte de sus superiores. Por su parte, la alta puntuación en doble presencia puede venir determinada por la falta de compaginación entre su vida laboral y familiar, ya que si como se menciona anteriormente, el horario es muy irregular y su carga de trabajo extensa, podría ocupar más horas de las lectivas, de ahí que no desconecte del trabajo y las exigencias psicológicas que perciba por parte de esta doble vida sean mucho más altas.

Por último, en lo que respecta a la dimensión de la estima, nuestro participante habla de una falta de valoración. Explica que como su trabajo depende del ministerio, tiene la sensación de que no se valora lo que hace. Al no haber ningún tipo de contacto directo con sus superiores, esta persona carece de un feedback positivo y reforzamiento que le haga sentirse capaz y satisfecho con el trabajo que realiza. Esto se traduce en una desgana y desilusión por el mismo, que también afecta, de forma indirecta a las exigencias psicológicas, pues acaba sintiendo que da (su trabajo) pero no recibe nada (excluyendo a lo económico).

Otra pieza clave para la explicación de su situación, parece ser la longevidad de su trabajo. Es decir, los años de antigüedad dentro del mismo sistema. Si una persona se encuentra dentro de una organización en la que las condiciones no son óptimas, con el paso de los años, el personal se verá cada vez más devaluado y superado por la situación. Quizás esto le suceda a nuestro participante, pues pasan los años, su situación no cambia y cada le cuesta más seguir en dicha tesitura.

\section{Referencias}

Branden, N. (2004). Cómo mejorar su autoestima. 14 edición. Barcelona: Paidós.

Branden, N. (1995). Los seis pilares de la autoestima. Paidós.

Branden, N. (1993). Poder de la Autoestima. Paidos Iberica, Ediciones S. A..

Durán, M. (2010). Bienestar Psicológico: el estrés y la calidad de vida en el contexto laboral. Revista Nacional de Administración, 1(1), 71-84.

Estevan, L., Rodríguez, R. M., Romero, B., Rodríguez, Á., \& Romo, M. (2014). Doble presencia: un riesgo psicosocial que evidencia la desigualdad entre hombres y mujeres en la conciliación de la vida familiar y laboral.

FOCUS on Young Adults (1998). Manual de capacitación para facilitadores. Mejorando habilidades y destrezas de comunicación interpersonal para la orientación a adolescentes en salud sexual y reproductiva. Lima: El Autor.

González-Pienda, J. A., Pérez, J. C. N., Pumariega, S. G., \& García, M. S. G. (1997). Autoconcepto, autoestima y aprendizaje escolar. Psicothema, 9(2), 271-289.

Instituto Sindical de Trabajo, Ambiente y Salud. (2003). Exceso de exigencias psicológicas. Recuperado el 17 de Abril de 2015, de http://istas.net/web/index.asp?idpagina=3937

ISTAS. (2002). Instrumento para la Prevención de Riesgos Psicosociales. http://pefc5.ugr.es/moodle/file.php/906/ISTAS-21_version_corta.pdf

Mauno, S.; Kinnunen, U.; Ruokolainen, M. (2007). Job demands and resources as antecedents of work engagement: A longitudinal study. Journal of Vocational Behavior, Vol. 70, n. 1, pp. 149-171. 
Molina, Margarita; Baldares; Thelma y Maya, Arnobio. (1996). Fortalezcamos la autoestima y las relaciones interpersonales. San José: Impresos Sibaja.

Moreno, L., Garcia, J. M., Díaz, M. J., \& Ramiro, E. (2005). Factores psicosociales en el entorno laboral. EduPsykhé,4(1), 19-42.

Moreno-Jiménez, B. \& Garrosa, E. (2013). Salud laboral. Riesgos psicosociales y bienestar laboral. Madrid: Ediciones Pirámide.

Rice, Philip. (2000). Adolescencia. Desarrollo, relaciones y cultura. Madrid: Prentice

Hall.

Sánchez, M. M. G., Prego, M. D. L. A. B., de la Campa Portela, R. M., \& Canosa, J. L. S. (2013). La doble presencia como riesgo psicosocial en las docentes universitarias. In Roles de xénero en tempos de crise (pp. 363-370). Servizo de Publicacións e Intercambio Científico.

Tarazona, D. (2014). Autoestima, satisfacción con la vida y condiciones de habitabilidad en adolescentes estudiantes de quinto año de media. Un estudio factorial según pobreza y sexo. Revista de Investigación en Psicología, 8(2), 57-65.

Undurraga, C. \& Avendaño, C. (1998). "Dimensión psicológica de la pobreza". Psykhe, 6(1), 57-63. 